\title{
Ultrastructural Studies in Fetal I-cell Disease
}

\author{
KAZUHIRO ABE, ICHIRO MATSUDA, SHINICHIRO ARASHIMA, ${ }^{(20)}$ TAKASHI MITSUYAMA, \\ YOGO OKA, AND MUTSUO ISHIKAWA \\ Departments of Anatomy, Pediatrics, and Obstetrics and Gynecology, Hokkaido University School of \\ Medicine, Sapporo, Japan
}

\section{Extract}

The skin, brain, lung, liver, and kidney from a 20 -week-old fetus who was diagnosed as having fetal I-cell disease by amniocentesis at 14 weeks of gestation were examined by light and electron microscopy. In addition, cultured fibroblasts from the skin were also observed microscopically. Cytoplasmic inclusions with dense poly morphic contents appeared commonly in the capillary endothelial cells in the skin, lung, glomerulus of the kidney, and the epithelial cells of the proximal tubules of the kidney, and sometimes in the hepatocytes of the liver and the nerve and glial cells of the brain. Erythropoietic cells in the liver and circulating erythrocytes contained dense inclusions varying in developmental stages. Fibroblasts of the skin had several clear vacuoles, and cultured fibroblasts were filled with dense inclusions. The dense cytoplasmic inclusions in fetal I-cell disease were light and electron microscopically similar to the residual bodies which are commonly observed in the phagocytic cells.

\section{Speculation}

In fetal I-cell disease, the cytoplasmic inclusions may first appear as dense bodies in the capillary endothelial cells of fetus as early as 4 weeks of gestation. Material stored in the inclusions may reflect deranged metabolism of the cells. Thus, the morphologic changes of I-cell disease may be due to the deficiencies of intralysosomal enzymes.

I-cell or inclusion cell disease associated with a Hurler-like syndrome, first described by Leory and De Mars in 1967 (6), is characterized by the presence of coarse cytoplasmic inclusions in cultured fibroblasts. By evidence from histochemical studies of the cytoplasmic inclusions, I-cell disease is classified as a type of mucopolysaccharidoses or mucolipidoses $(6,15)$. The morphologic alterations of this inborn disease seemed to appear in the early prenatal stages, but little information is available about morphologic studies of fetal I-cell disease except for very recent ultrastructural observations by Aula et al. (1). This article also deals with ultrastructural observations of various tissues and cultured skin fibroblasts from a fetus with I-cell disease. In the previous ultrastructural studies in children with this disease, round cytoplasmic vacuoles with a clear matrix were usually demonstrated $(5,14)$ and the vacuoles filled the cytoplasm. From such findings, it is difficult to know the early configuration of the inclusions and its relation to the cell organelles. Our consideration is that ultrastructural investigations of fetal tissues in I-cell disease may show the early developmental stages of the inclusions. In the present studies, the finding that cells in various tissues contained various types of cytoplasmic inclusions with dense contents indicated a possible developmental process of the inclusions. The findings may be useful for understanding of the nature and pathogenesis of cytoplasmic inclusions in I-cell disease. The clinical and biochemical examinations of the amniotic fluid and
I-cells from the present fetus were described in separate papers $(9,11)$.

\section{MATERIALS AND METHODS}

A pregnancy of a 21-year-old mother from a family at risk for I-cell disease was monitored and the diagnosis of the disease was made by biochemical examination of the amniotic fluid and cells obtained at 14 weeks of gestation (9). The pregnancy was interrupted by therapeutic abortion at 20 weeks of gestation. The aborted fetus was male, $160 \mathrm{~g}$ in weight, and revealed macroscopically normal development corresponding to the fetal age.

Immediately after the abortion, tissue fragments of the abdominal skin, brain, lung, liver, and kidney from the fetus were immersed in $5 \%$ formol in $0.05 \mathrm{M}$ phosphate buffer $(\mathrm{pH} 7.5)$ containing $1.5 \%$ sucrose. For light microscopic studies, the tissues were fixed for $48 \mathrm{hr}$ and paraffin sections were stained with periodic acid-Schiff reagent (PAS) and hematoxylin. The sections were generally examined after digestion of glycogen with diastase, because abundant glycogen covered other PAS-positive substance in the tissues. For electron microscopic studies, the tissues were fixed in formol for $36 \mathrm{hr}$, postfixed in $2 \% \mathrm{OsO}_{4}$ in $0.05 \mathrm{M}$ phosphate buffer ( $\mathrm{pH} 7.5$ ) containing $1.5 \%$ sucrose for $2 \mathrm{hr}$, and embedded in Epon. Ultrathin sections stained with uranyl acetate and lead citrate were examined under a Hitachi electron microscope (HS-9).

Skin fibroblasts from the fetus were cultured in monolayer on a siliconized cover glass by a procedure reported previously (10). On the 4 th day of the 2 nd subculture, the monolayer was fixed in $5 \%$ formol for $20 \mathrm{hr}$ and the cover glass with the monolayer was cut into three pieces. Two pieces were stained with PAS-hematoxylin and Sudan black B-hematoxylin for light microscopic studies. Another was postfixed in $2 \% \mathrm{OsO}_{4}$ for $1 \mathrm{hr}$ and the monolayer was embedded in Epon for electron microscopic studies. Semithin sections of Epon-embedded tissues or cultured cells were stained with toluidine blue for light microscopy.

\section{RESULTS}

\section{SKIN}

The epithelial cells in the epidermis and hair follicles rarely contained PAS-positive granules. Most of the fibroblasts were filled with numerous vacuoles. Cells containing a varying number of fine PAS-positive granules appeared rarely in the skin connective tissue. Some few cells had both vacuoles and PAS-positive granules. All of the capillary endothelial cells contained numerous PAS-positive granules.

In electron microscopic studies, all fibroblasts of the skin contained several clear, round vacuoles, $0.5-1.0 \mu \mathrm{m}$ in diameter, which were scattered in the cytoplasm except in the Golgi area (Fig. 1, $a$ and $b$ ). The vacuoles included small numbers of tortuous microtubules, $20 \mathrm{~nm}$ in diameter. They sometimes contained a few 

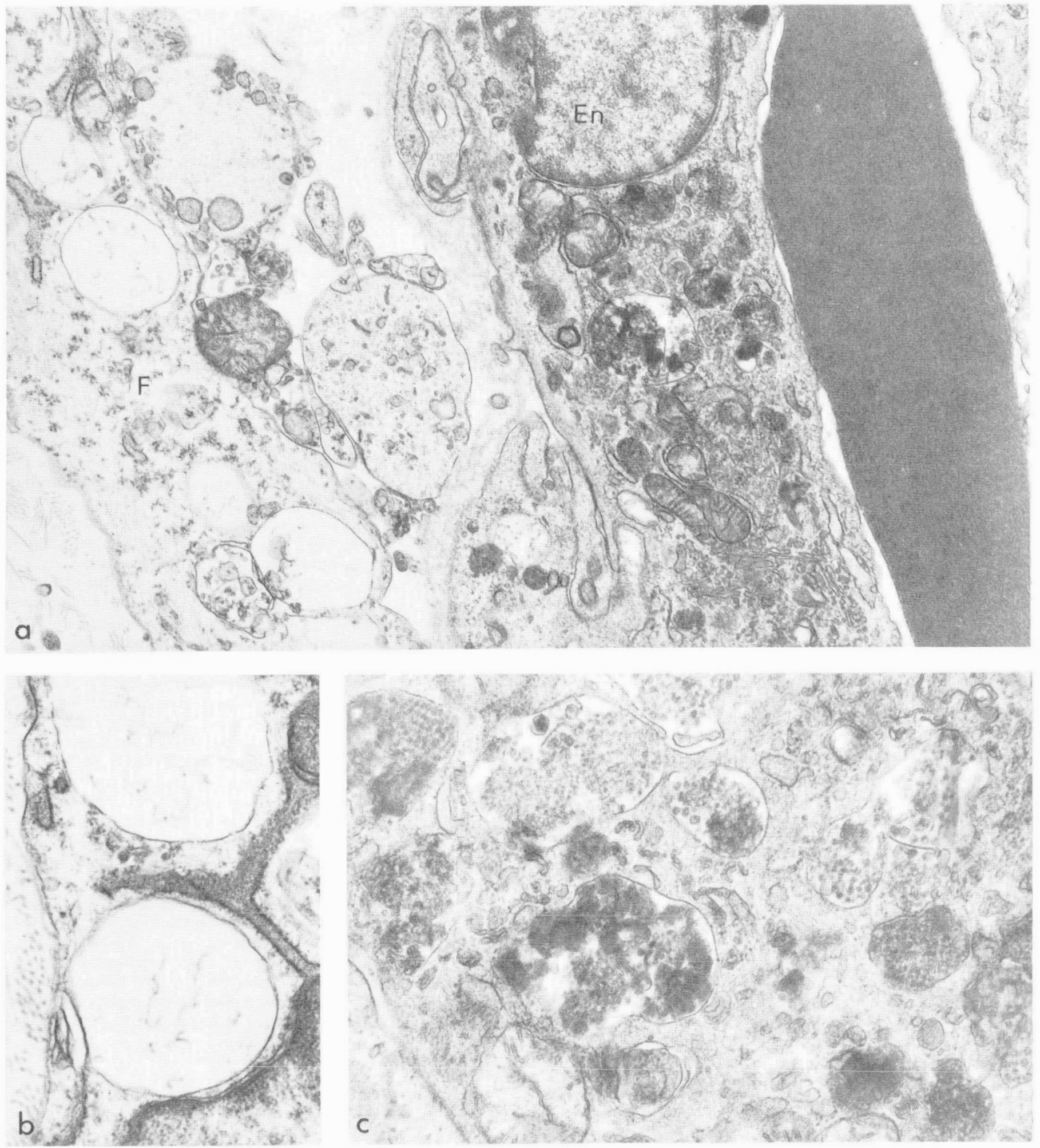

Fig. 1. Skin. $a$ : a fibroblast $(F)$ containing clear vacuoles and a endothelial cell $(E n)$ containing dense inclusions $(\times \quad 15,000) ; b$ : clear vacuoles in the fibroblast include fine tortuous tubules $(\times \quad 36,000) ; c$ : dense inclusions in the endothelial cell contain fine vesicles and clumped materials of variable sizes $(\times 32,000)$.

membranous elements. In addition to clear vacuoles, rare fibroblasts contained inclusions with dense polymorphic contents Macrophages occurred rarely. These contained lysosomal bodies and inclusions with polymorphic contents. All of the endothelial cells of the capillaries contained numerous membrane-bound inclusions, $0.2-1.0 \mu \mathrm{m}$ in diameter (Fig. 1, $a$ and $c$ ). The inclusions were round or irregular in shape and were generally filled with fine vesicles $20-40 \mathrm{~nm}$ in diameter. They often contained dense, clumped materials varying in size and shape. Membranous elements and myelin figures were rarely observed in the inclusions. The inclusions were distributed throughout the cytoplasm. They were especially frequent near the Golgi area.

BRAIN

Cells with PAS-positive granules were rarely found in the brain. By electron microscopy, the brain was still undifferentiated, and 
the nerve and glial cells were immature. Most of the cells had no inclusions, although a few contained several inclusions which were similar in features to those in the capillary endothelial cells of the skin. However, no inclusions appeared in endothelial cells of the capillaries in the brain.

LUNG

The lung consisted of epithelial tubules embedded in the abundant stroma. Neither epithelial cells nor stroma cells contained PAS-positive granules. The PAS-positive granules were seen in the capillary endothelial cells, but they were fewer in the lung than in the skin.

Electron microscopic studies revealed that the stroma was composed of undifferentiated mesenchymal cells containing abundant glycogen. Fibroblasts with vacuoles or inclusions were not observed. The immature endothelial cells forming accumulations of several cells with narrow capillary lumen also contained dense inclusions similar to those of capillary endothelial cells in the skin.

\section{LIVER}

In the liver hepatocytes generally contained small numbers of PAS-positive granules, although some hepatocytes grouped in various regions of the liver contained numerous PAS-positive granules (Fig. 2a). The PAS-positive granules in hepatocytes were wine red, whereas PAS-positive granules were pinkish red in capillary endothelial cells in the skin and lung. Such wine red granules in hepatocytes were accumulated in the cytoplasm along the bile canaliculi and along the border facing the interlobular connective tissue. Kupffer's cells also contained pink granules of varying size. Fibroblasts in the interlobular connective tissue did not have PAS-positive granules.

By electron microscopy most of the hepatocytes contained no inclusions, but some hepatocytes possessed several inclusions, $0.2-1.0 \mu \mathrm{m}$ in diameter, which had moderately dense, amorphous, or finely granular matrix and flecked dense cloudy substance (Fig. $2, b$ and $c$ ). In addition, some inclusions, like normal lysosomal bodies, contained granules, vesicles, and membranous elements. The inclusions were generally present around the Golgi apparatus near the bile canaliculi. Kupffer's cells contained dense lysosomal inclusions and polymorphic inclusions such as seen in the capillary endothelium. A few interstitial cells in perisinusoidal spaces possessed several clear vacuoles, $0.5-1.5 \mu \mathrm{m}$ in diameter, containing several small vesicles and occasional fat droplets.

In the interlobular connective tissue, fibroblasts contained a few clear vacuoles, and endothelial cells of the blood vessels often contained dense inclusions. Some of the epithelial cells of the terminal bile ducts contained inclusions similar to those in the hepatocytes. On the other hand, granular myelocytes and leucocytes had no abnormal inclusions.

\section{KIDNEY}

In the kidney, development of various constituents of the nephrons was in progress. In both the newest glomeruli just below the capsule and in more developed glomeruli in the deeper region, numerous PAS-positive granules appeared in the endothelial cells but not in the epithelial cells (Fig. 3a). In the epithelial cells of the proximal tubule, the luminal border was stained positively with PAS and numerous pink granules appeared around the nucleus. The epithelial cells of the distal tubule generally contained no PAS-positive granules. The epithelial cells of the collecting tube contained several PAS-positive granules in the apical cytoplasm. The stroma histologically resembled that of the lung.

By electron microscopy glomerular endothelial cells contained numerous inclusions, and they were similar in features to capillary endothelial cells in the skin (Fig. 3b). Even endothelial cells of the less differentiated glomeruli contained similar inclusions. The epithelial cells of the renal corpuscles had no inclusions. The epithelial cells of the proximal tubule already showed apical tubular invaginations, and contained inclusions, $0.2-1.5 \mu \mathrm{m}$ in diameter, which were similar in feature to the inclusions in hepatocytes and capillary endothelial cells. The inclusions contained a dense, cloudy substance and fine vesicles (Fig. 3, $c$ and $d$ ). In addition, some inclusions had parallel lamellas.

\section{ERYTHROPOIETIC CELLS AND ERYTHROCYTES (FIG. 4}

The liver contained numerous erythropoietic cells (Fig. 2a). Erythropoietic cells in the liver and erythrocytes in circulating blood contained several PAS-positive granules. In erythrocytes, a few vacuoles, often including PAS-positive granules, appeared in the cytoplasm (Fig. 4e).

By electron microscopy erythropoietic cells at various developmental stages in the liver contained a few or several inclusions containing amorphous dense substance, dense granules or clumps of varying size, membranous elements, or fine vesicles (Fig. 4, $a-d)$. Proerythroblasts or young erythroblasts had a few inclusions around Golgi apparatus. The inclusions were filled only with minute vesicles. Erythrocytes had inclusions which appeared as vacuoles with polymorphic dense clumped or membranous contents. The inclusions in the erythroblasts or erythrocytes, like siderosomes, are often associated with ferritin particles (Fig. $4 d$ ).

\section{CULTURED SKIN FIBROBLASTS}

Cultured fibroblasts were spindle shaped or more angular with a few cytoplasmic processes (Fig. 5, $a$ and $b$ ). The nuclei were oval in shape, varying in size, and had several nucleoli. The cytoplasm was filled with numerous granular inclusions. The inclusions were absent in an area adjacent to the nucleus and scanty in the cytoplasmic processes. Histologically the inclusions were of two types. In type 1 , most of the inclusions were stained weakly or moderately with PAS, weakly or not at all with Sudan black B, and moderately or strongly with toluidine blue. In type 2, some of the inclusions were stained almost negatively with PAS, strongly with Sudan black B, and negatively with toluidine blue. All of the inclusions showed no metachromasia in toluidine blue staining. The inclusions were also observed in mitotic cells. Some fibroblasts had cytoplasmic fibrils which were stained weakly with PAS and glycogen.

In electron microscopic studies, the fibroblasts were different in ultrastructure from fibroblasts in the skin, and they had a large clear nucleus with large nucleoli (Fig. $5 c$ ). The cytoplasm was filled with numerous inclusions, $0.2-1.8 \mu \mathrm{m}$ in diameter, which were round, oval, or irregular in shape. The inclusions had moderately dense or dense polymorphic contents which consisted of amorphous substance, granules and clumps of varying size, fine vesicles, and membranous elements (Fig. 5, $c$ and $d$ ). Several densely osmiophilic lipid granules, $0.2-1.0 \mu \mathrm{m}$ in diameter, small rough endoplasmic reticulum, and mitochondria were scattered among the inclusions. The mitochondria were long and branched in shape. The Golgi apparatus was well developed, but no inclusions were seen in the Golgi area. The peripheral wide region of the cytoplasm lacked inclusions and had abundant glycogen in addition to long tortuous mitochondria and vesicular smooth endoplasmic reticulum, lacking inclusions.

\section{DISCUSSION}

I-cell disease is one of the inborn lysosomal diseases, and its pathologic changes are known to be characterized by the presence of numerous cytoplasmic inclusions in both various tissues and cultured fibroblasts in affected children $(6,14,16)$. The present findings demonstrated that characteristic cytoplasmic inclusions were contained in various types of cells in an affected 20-week-old fetus. Inclusions were particularly prevalent in capillary endothelial cells in various tissues; even primitive forms of the endothelial cells had inclusions. However, no inclusions were seen in undifferentiated mesenchymal cells.

Embryologically, the primitive endothelium is differentiated from mesenchymal cells, known as angioblasts. Endothelial cells seemed to contain the inclusions as a functional differentiation from mesenchymal cells, although the reason for this difference is 

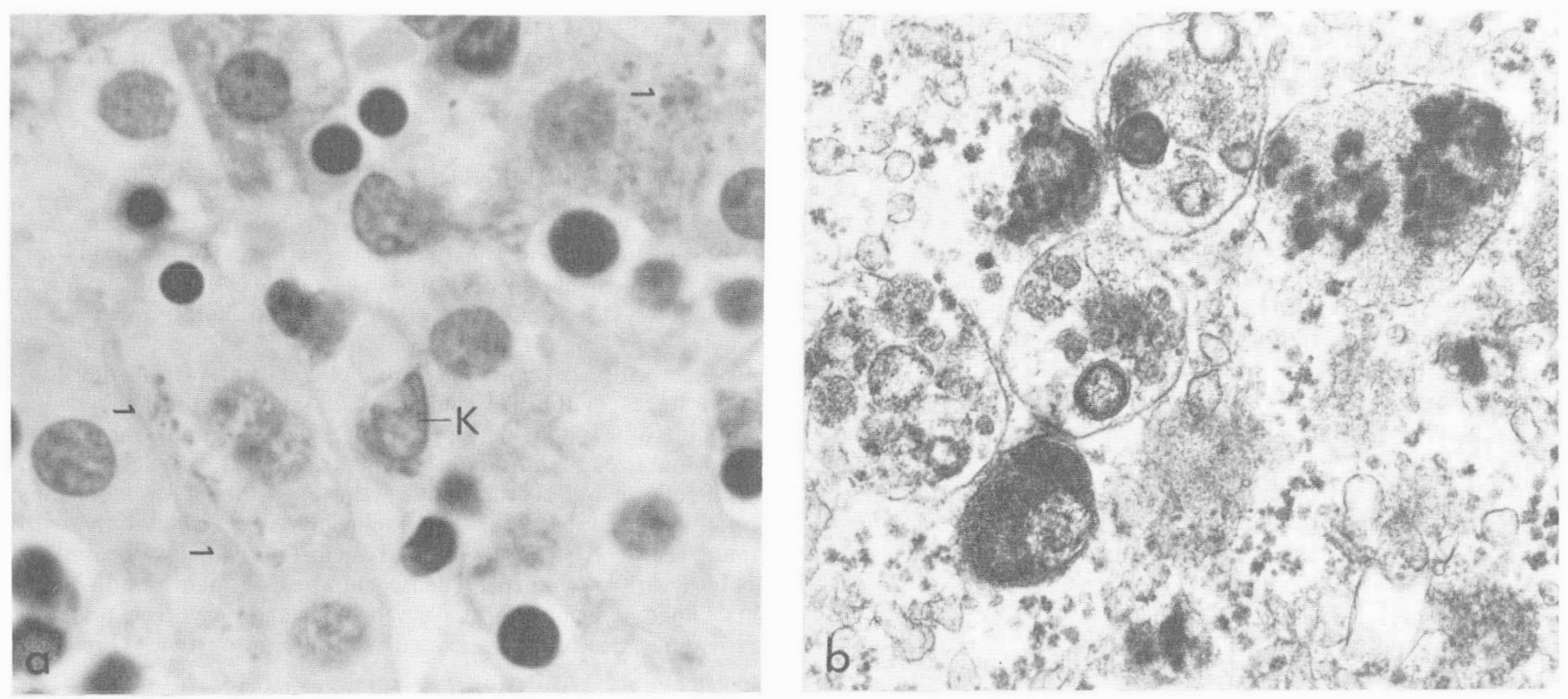

5.

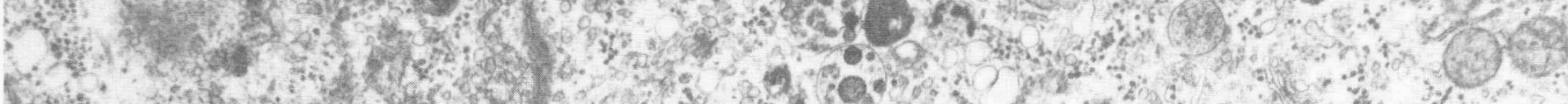

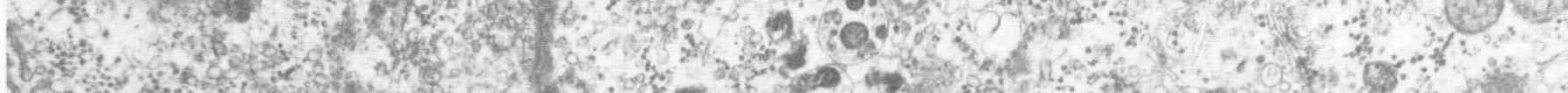

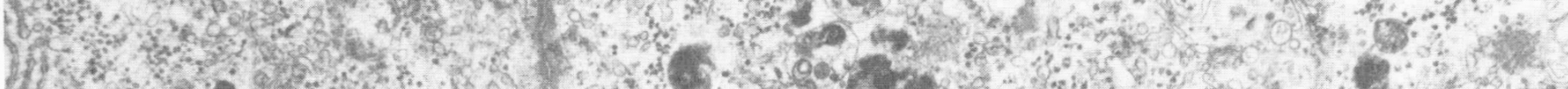
ff

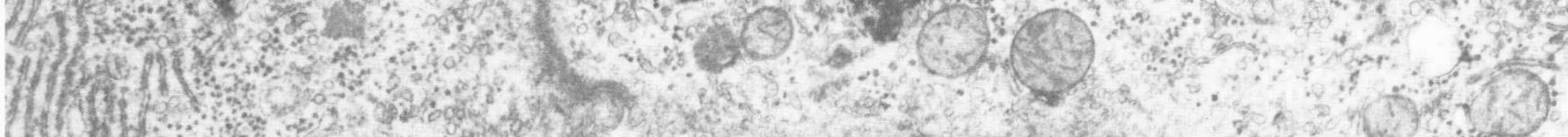
4 (IIf

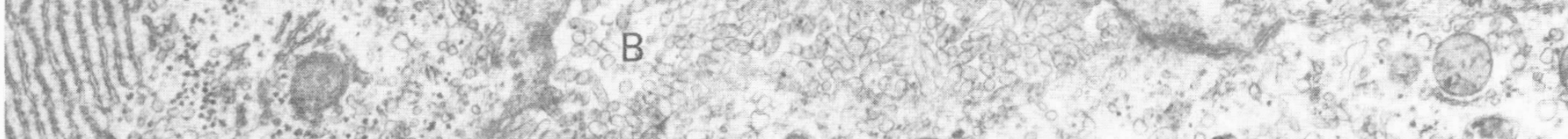

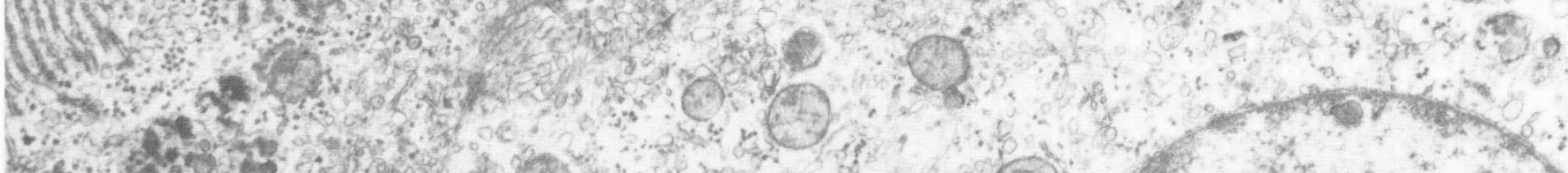
2y

\section{(x)}

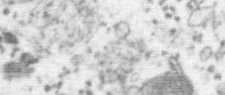

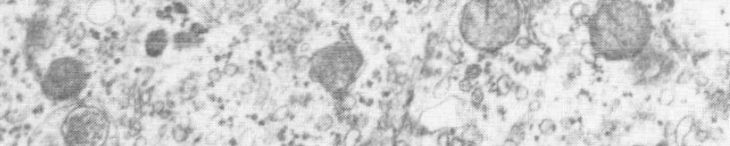

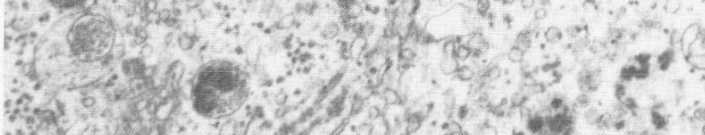
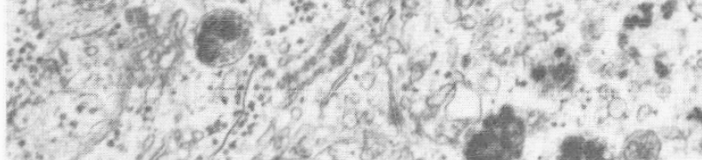

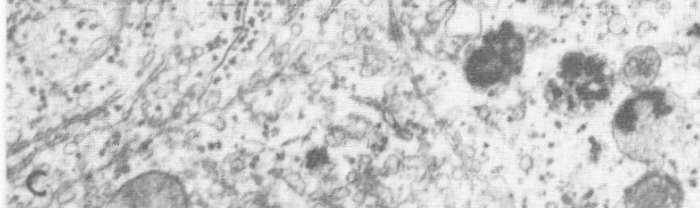

Fig. 2. Liver. $a$ : some hepatocytes contain dense periodic acid-Schiff (PAS)-positive granules along bile canaliculi (arrows). A Kupffer cell (K) contains PAS-positive granules. Many erythroblasts are visible $(\times 1,400)$. $b$ : inclusions in a hepatocyte. Lysosomal bodies and inclusions with an amorphous matrix and flecked dense cloudy substance are shown $(\times 18,000) . c$ : hepatocytes. Dense cytoplasmic inclusions appear around the bile canaliculus, $B$ $(\nvdash 10,000)$

unknown. In the embryo, the cardiovascular system is the first system to function and appears as early as 3-4 weeks of gestation. Thus, capillary endothelium is differentiated in so early an embryonic stage that the inclusions may appear from the early stage of gestation. Therefore, diagnosis of this disorder may be possible in stages of gestation prior to 14 weeks, when the present case was diagnosed as I-cell disease by amniocentesis.

The ultrastructural features of inclusions varied in different types of cells. Ultrastructurally, inclusions of three types were identified. Type $l$ was characterized by clear inclusions or 

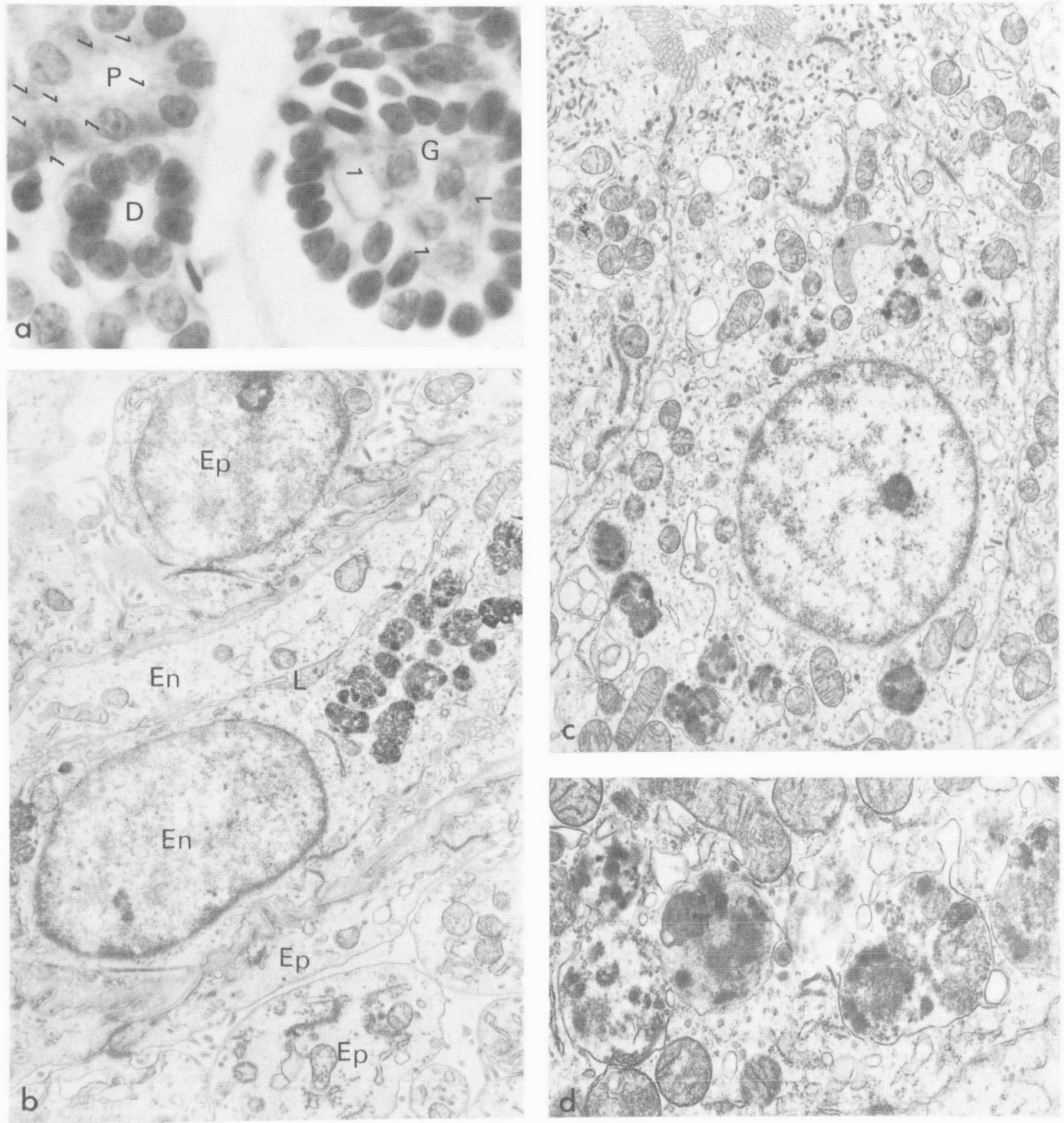

Fig. 3. Kidney. $a$ : in a primitive glomerulus $(G)$ periodic acid-Schiff (PAS)-positive granules (arrows) are visible in the endothelial cells. Numerous PAS-positive granules (arrows) are present in epithelial cells of the proximal tubule $(P)$, but are absent in epithelial cells of the glomerulus and distal tubule, $D(\times 1,200), b$ : glomerulus. Primitive endothelial cells $(E n)$ with narrow lumen $(L)$ contain dense inclusions. Epithelial cells $(E p)$ contain no inclusions $(\times 9,000)$. $c$ : numerous dense inclusions appear around the nucleus in an epithelial cell of the proximal tubule. Dense tubular invaginations and short microvilli are visible, upper $(\times 12,000) . d$ : inclusions in the epithelial cell of the proximal tubule have fine vesicles, clumps, and cloudy substance $(\times 22,000)$.

vacuoles, which appeared in fibroblasts in the skin and liver. Type 2 had dense inclusions containing numerous fine vesicles and occasional granular and clumped substances. These types of inclusions appeared most frequently. They were seen in capillary endothelial cells, erythropoietic cells, and, sometimes, in cells of the brain. Inclusions in cultured skin fibroblasts showed more advanced features. They contained more numerous coarse granu- lar and clumped substances. Type 3 was marked by dense inclusions containing cloudy flecked substance in the diffuse matrix. This type of inclusions appeared in hepatocytes. Inclusions in the epithelial cells of the renal proximal tubule revealed intermediate features of type 2 and type 3 . This may be explained in relation to the fact that both hepatocytes and epithelial cells of the renal tubule have peroxisomes. 

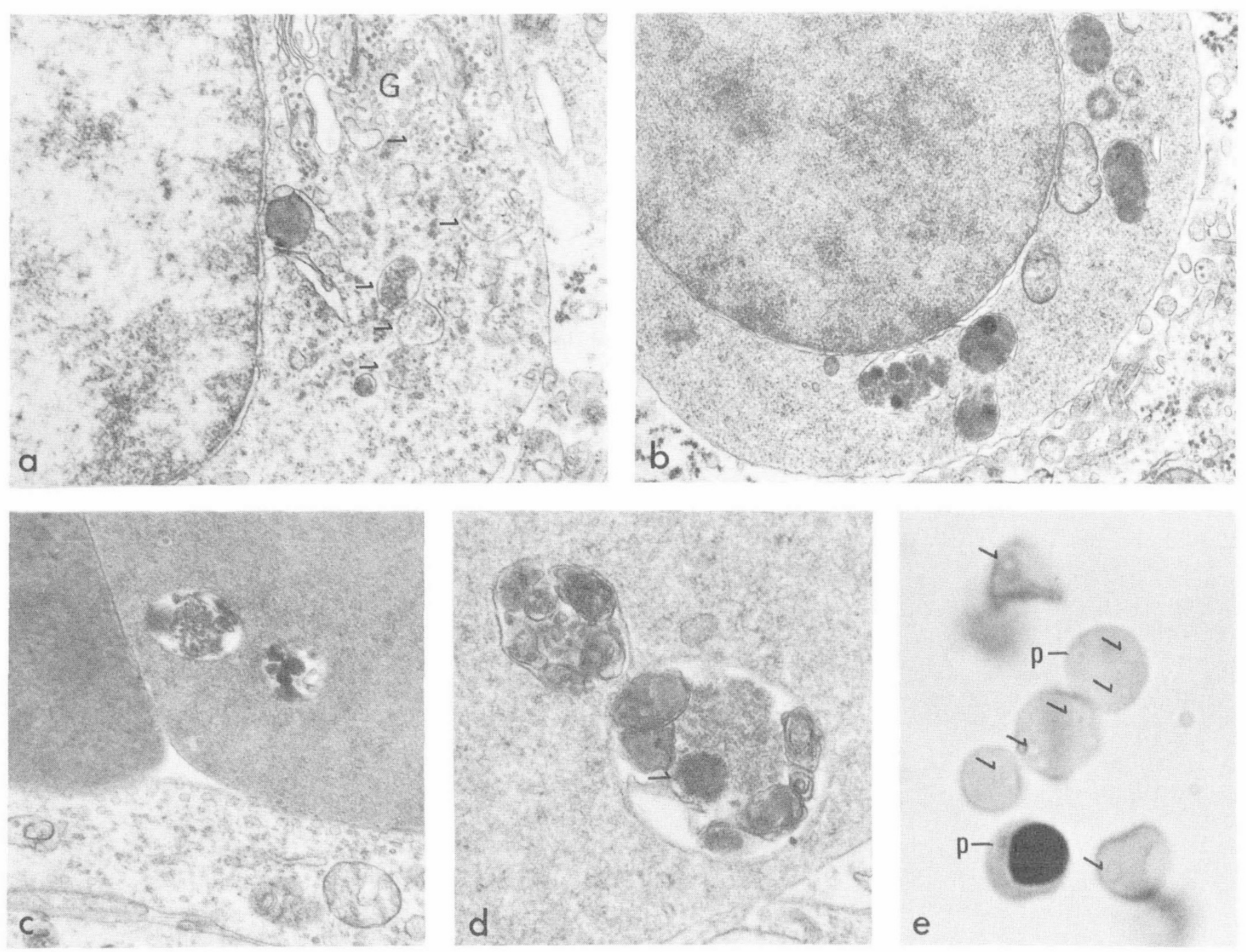

Fig. 4. Erythropoietic cells in various developmental stages and erythrocytes. $a$ : proerythroblast. Inclusions (arrows) filled with fine vesicles are seen around the Golgi apparatus, $G(\times 26,000)$. b: erythroblast with inclusions containing dense amorphous substance and fine vesicles $(\times 18,000)$. $c$ : inclusions in an erythrocyte containing polymorphic materials $(\times 18,000) . d$ : inclusions in a erythrocyte containing granules, vesicles, clumps, and membranous elements. An inclusion has an accumulation of ferritin particles, arrow ( $\times$ 40,000). e: vacuoles (arrows) and periodic acid-Schiff-positive granules $(p)$ are seen in erythrocytes and an erythroblast $(\times 2,000)$.

The three types of inclusions are diagrammatically shown in Fig. 6. The morphologic heterogeneity of inclusions may reflect different lysosomal activities in different cells. The frequency of appearance of inclusions varied in different tissues and cells, but the types of inclusions were generally similar in each type of cell. Thus, most of the inclusions were of the dense type.

A quantitative assessment of the types of inclusions in different tissues and cells may be required for comparison between morphologic and biochemical analyses, but it is difficult to make sampling and measurement for precise quantitative analysis. The dense inclusions resemble lysosomal residual bodies in appearance. As is generally known, residual bodies, containing sudanophilic and PAS-positive substances, are often observed in phagocytotic cells. The bodies are considered to be materials incompletely digested because of physiologic deficiencies of intralysosomal enzymes (4). Such cytoplasmic inclusions have also been reported to appear in granular cell tumor cells $(2,12)$ and degenerating cells $(4,13)$. In I-cell disease showing pathologic deficiencies of intralysosomal enzymes, however, residual body-like structures are numerous in cells which have no or few inclusions in normal tissues.

Although the cause of deficiencies of intralysosomal enzymes in I-cell disease is still under discussion, recent investigations have suggested that genetic disorders of lysosomal functions may arise from excessive loss of lysosomal enzyme into extracellular fluid because of possible structural defects of these enzymes (16). We evaluated this disease in this respect by biochemical studies $(10,11)$.

In the present investigation, it was found that erythrocytes in the prenatal stage contained several inclusions, although no reports are available which state that erythrocytes in patients with I-cell disease contain these inclusions. Furthermore, in spite of additional investigations, we were unable to find such inclusions in the peripheral erythrocytes in the sibling of this fetus and in a child with this disorder. Fetal and postnatal erythroblasts produce different hemoglobins. Fetal erythroblasts may be more strongly influenced by certain affected enzymes.

Erythropoietic cells in various developmental stages were seen in the liver. Between the proerythroblast and the mature erythrocyte is a series of developmental stages, characterized well with electron microscopy. We can assume morphologic development of inclusions, if the configurations of inclusions in the erythropoietic cells are arranged in order of the stages of erythropoiesis. Thus, inclusions in erythroblasts and erythrocytes revealed a series of morphologic changes of development. Proerythroblasts and young erythroblasts had a few inclusions around the Golgi apparatus. The inclusions were filled only with fine vesicles, and they appeared to be multivesicular bodies, one type of lysosome $(3,8)$. The present 

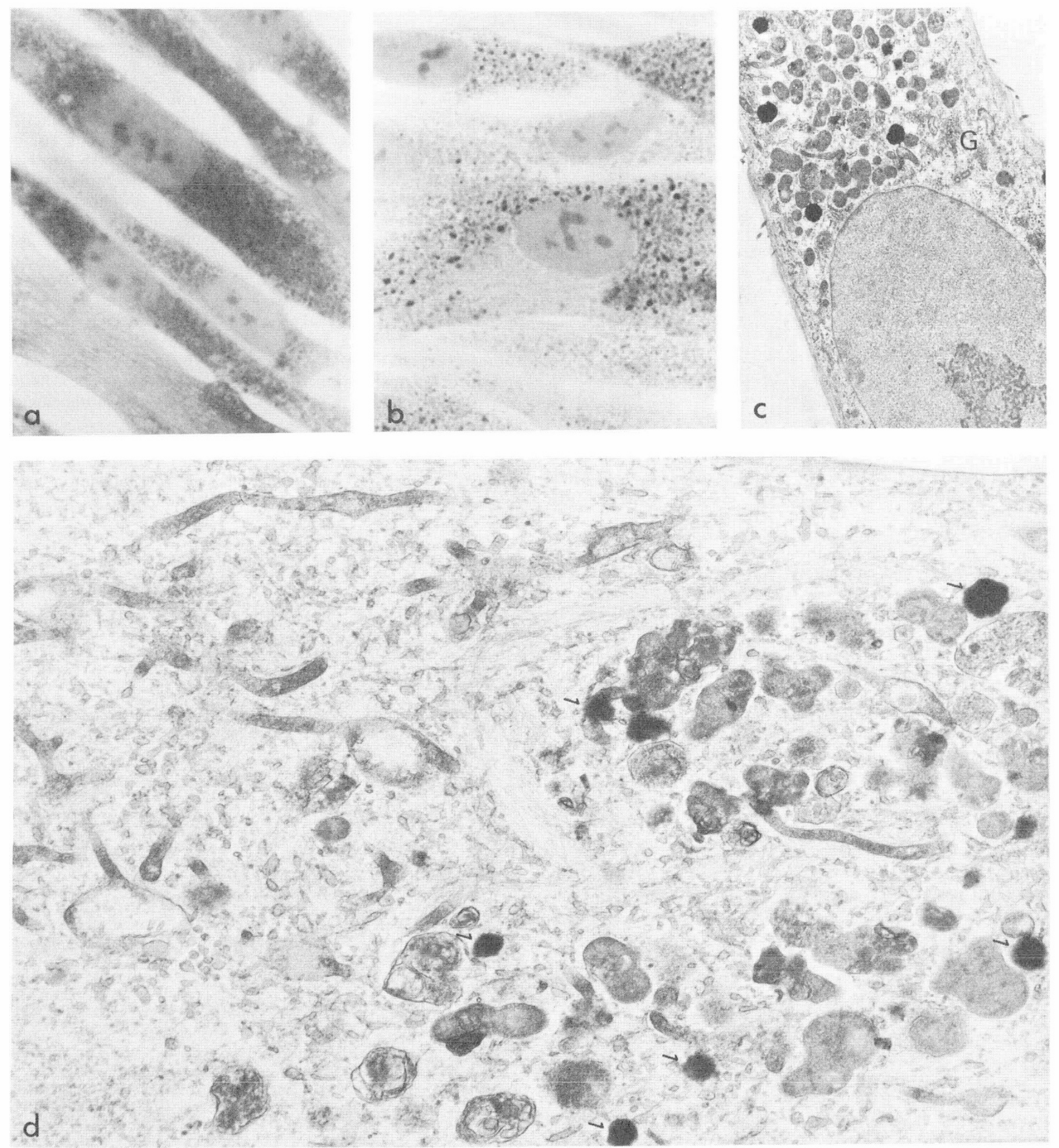

Fig. 5. Cultured skin fibroblasts. $a$ : the cells are filled with granules varying in the intensity of periodic acid-Schiff staining $(\times \quad 1,000)$. $b$ : in Sudan black staining, most granules are stained weakly, but some are stained strongly. An area devoid of inclusions is close to the nucleus in a fibroblast ( $\times 1,000) . c$ : a fibroblast is filled with dense inclusions except for the Golgi area, $G(\times \quad 6,000)$. $d$ : cytoplasm of a fibroblast. Inclusions contain granular, fine vesicular, clumped and membranous contents. Several lipid granules are scattered throughout (arrows). In the area devoid of inclusions (left), long mitochondria with tortuous branches, vesicular smooth endoplasmic reticulum, and fine filaments are visible $(\times 15,000)$.

observations of the inclusions in erythropoietic cells in various stages of development suggest that the inclusions are formed around the Golgi apparatus and that contents of the inclusions undergo a series of morphologic changes. As shown in Figure 7, contents seemed to change from fine vesicles to fine granules and dense clumps and to develop into polymorphic large dense clumps and membranous elements among amorphous dense substance.
According to previous investigations in postnatal patients with I-cell disease, the inclusions generally consisted of clear vacuoles $(5,14)$. As seen in the present findings, however, the inclusions generally contained dense contents. Clear vacuoles containing peculiar microtubules appeared in skin fibroblasts, but they were infrequent in other cells. It was believed that clear vacuoles in I-cell disease had a lysosomal nature, because the transformation of the 


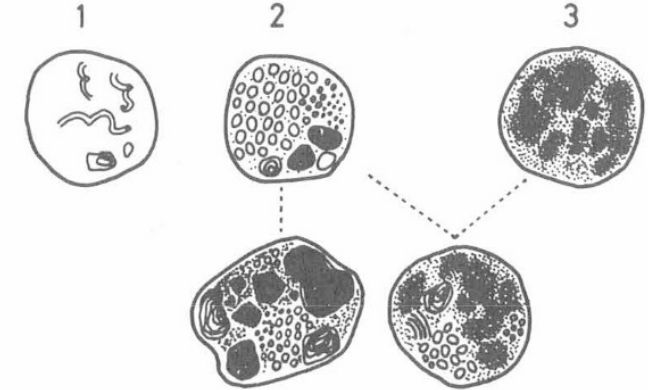

Fig. 6. Three types of inclusions appeared in cells in various tissues (upper). Lower two show inclusions in cultured fibroblasts (left) and epithelial cells of the renal proximal tubule (right).

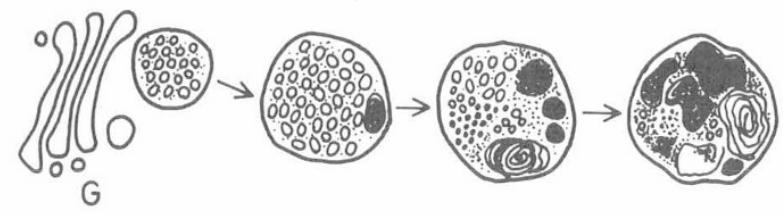

Fig. 7. A possible developmental process of the most common type (type 2) of inclusion. G: Golgi apparatus.

dense lysosomal bodies of hepatocytes was previously shown to occur in rats and mice after the injection of various polysaccharides which are stored in lysosomes (15). In the present case, the contents of clear vacuoles were negative in PAS staining, and intermediate types, from dense inclusions to clear vacuoles, were not observed in fibroblasts in the tissue. From these findings, clear vacuoles in fibroblasts in the fetus do not appear to be formed by transformation from dense bodies. Cultured fibroblasts, however, were filled with dense inclusions, although they were significantly different in cytologic features from fibroblasts in situ. Therefore, the lysosomal enzyme activities may be different between fibroblasts in situ and fibroblasts in vitro. Cultured fibroblasts contained dense inclusions even during cell division. Thus the inclusions seem to be carried into daughter cells in mitotic division.

It had been observed that dense inclusions in cultured fibroblasts were transformed into clear vacuoles as repeated subcultures (15). In the present case, capillary endothelial cells, hepatocytes, and epithelial cells of renal tubules, which were filled with clear vacuoles in postnatal periods, contained only dense inclusions. The relation between the dense inclusions in the fetus and clear vacuolar inclusions found in affected children is yet obscure, although it may not be denied that the dense inclusions were gradually transformed into clear vacuoles during repeated cell generations.

In I-cell disease it has been accepted that enzymes for hydrolysis of glycoproteins or glycolipids are deficient in lysosomes and that, subsequently, glycoproteins or glycolipids, stained with PAS, Sudan, or Alcian blue are deposited in the lysosomes $(5,14)$. It is believed also that morphologic differences of the inclusions are due to the nature of deposited substances depending on certain affected lysosomal enzymes. The biochemical analysis of lysosomal enzymes in the present case showed that activities of hexosaminidase, $\alpha$-galactosidase, $\beta$-galactosidase, $\beta$-glucuronidase, $\alpha$-fucosidase, and $\alpha$-mannosidase were significantly reduced and acid phosphatase was within a normal range in cultured skin fibroblasts (9). In the liver from the fetus, however, only the activity of $\beta$-galactosidase was deficient (11), as reported in the postnatal liver tissue by previous investigators (7). In respect to these biochemical findings, it is interesting that the ultrastructural types of inclusions showed differences between cultured skin fibroblasts and hepatocytes. However, biochemical analysis of affected enzymes and deposited substances in cells may not explain the different appearance of the inclusions, since various types of cells are contained in the tissues and they differ in metabolism.

\section{SUMMARY}

The skin, brain, lung, liver, and kidney from a 20-week-old fetus who was diagnosed as having fetal I-cell disease by amniocentesis at 14 weeks of gestation and cultures of fibroblasts from the skin were examined by light and electron microscopy. PAS-positive cytoplasmic inclusions appeared in various tissues. Ultrastructurally, type $l$ inclusions, or vacuoles appeared in fibroblasts in the skin and liver; type 2 or dense inclusions containing vesicles, granules, and clumps appeared in capillary endothelial cells, cultured fibroblasts, erythropoietic cells, and sometimes in cells of the brain; and type 3 or dense inclusions containing cloudy, flecked substance in the diffuse matrix were seen in hepatocytes. Inclusions in epithelial cells in the renal proximal tubule showed intermediate features of types 2 and 3 . Inclusions of type 2 were most frequent in various tissues, especially in capillary endothelial cells, and appeared even in primitive cells of this kind. Erythropoietic cells in various stages of differentiation showed a series of morphologic changes of the inclusions.

The results suggest that the inclusions appear in capillary endothelial cells in early embryonic stages and that they are produced as multivesicular bodies around Golgi apparatus and develop into inclusions with polymorphic contents, like residual bodies.

\section{REFERENCES AND NOTES}

1. Aula, P., Rapola, J., Autio, S., Raivio, K., and Karjalaninen, O.: Prenatal diagnosis and fetal pathology of I-cell disease (mucolipidosis type II). J. Pediat., 8: 221 (1975).

2. Fisher, E. R., and Wechsler, H.: Granular cell myoblastoma-a misnomer: Electron microscopic and histochemical evidence concerning its Schwann cell deviation and nature (granular cell schwannoma). Cancer, 15: 936 (1962).

3. Friend, D. S.: Cytochemical Staining of multivesicular body and Golgi vesictes. J. Cell Biol., 41: 269 (1969).

4. Hers, H. G.: The concept of inborn lysosomal disease. In: H. G. Hers and F. Van Hoof: Lysosomes and Storage Disease, p. 147 (Academic Press, New York, 1973).

5. Kenyon, K. R., Senenbrenner, J. A., and Wyllie, R. G.: Hepatic ultrastructure and histochemistry in mucolipidosis II (I-cell disease). Pediat. Res., 7: 560 (1973).

6. Leory, J. G., and De Mars, R. I.: Mutant enzymatic and cytological phenotypes in cultured human fibroblasts. Science, 157: 804 (1967).

7. Leory, J. G., Wan Ho, M., Mac Brinn, M. C., Zielke, K., Jacob, J., and O'Brien, J. S.: I-cell disease: Biochemical studies. Pediat. Res., 6: 752 (1972)

8. Martin, B. J., and Spicer, S. S.: Multivesicular bodies and related structures of the syncytio trochoblast of human term placenta. Anat. Rec., 175: 15 (1973).

9. Matsuda, I., Arashima, S., Mitsuyama, T., Kaneko, Y., and Ishikawa, M.: Prenatal diagnosis of I-cell disease. Humangenetik., 30: 69 (1975).

10. Matsuda, I., Arashima, S., Oka, Y., Mitsuyama, T., Ariga, S., Ikeuchi, T., and Ichida, T.: Prenatal diagnosis of fucosidosis. Clin. Chim. Acta, 63: 55 (1975).

11. Mitsuyama, T., Matsuda, I., Arashima, S., Oka, Y., and Homma, M.: To be published.

12. Moscovitz, E. A., and Azar, H. A.: Multiple granular cell tumors (myoblastomas): Case report with electron microscopic observations and review of literature. Cancer, 20: 2032 (1967).

13. Réne, A. A., Praden J. H., and Parker, J. L.: Radiation induced ultrastructural and biochemical changes in lysosomes. Lab. Invest., 25: 230 (1971).

14. Tondeur, M., Vamos-Hurwitz, E., Mockei-Pohi, S., Dereume, J. P., Cremer, N., and Loeb, H.: Clinical, biochemical and ultrastructural studies in a case of chondrodystrophy presenting the I-cell phenotype in tissue culture. J. Pediat., 73: 366 (1971)

15. Van Hoof, F.: Mucopolysaccharidoses. In: H. G. Hers and F. Van Hoof: Lysosomes and Storage Disease, p. 228 (Academic Press, New York, 1973).

16. Wiesman, U. N., and Herschkowitz, N. N.: Studies on the pathogenic mechanisms of I-cell disease in cultured fibroblasts. Pediat. Res., 8: 865 (1974).

17. We wish to thank Dr. Takashi Ito of the Department of Anatomy, Hokkaido University School of Medicine, for suggestions and critical review of the manuscript.

18. The present address of Dr. I. Matsuda: Department of Pediatrics, Kumamoto University School of Medicine, Kumamoto (Japan).

19. This work was supported by a research grant aided by the Ministry of Health and Welfare of Japan for the Research on Handicapped Children, 1975.

20. Requests for reprints should be addressed to: S. Arashima, M.D., Department of Pediatrics, Hokkaido University School of Medicine, Sapporo (Japan).

21. Accepted for publication January 28, 1976 